\title{
Causal Relationship of Overweight/Obesity on Cardiovascular Events Through Risk Factors: Multiple Mediation Analysis
}

Thosaphol Limpijankit ( $\nabla$ thosaphol.lim@mahidol.ac.th )

Ramathibodi Hospital

Prin Vathesatogkit

Ramathibodi Hospital

Dujrudee Matchariyakul

Electricity Generating Authority of Thailand

Sirichai Wiriyatanakorn

Ramathibodi Hospital

Sukanya Siriyotha

Ramathibodi Hospital

Ammarin Thakkinstian

Ramathibodi Hospital

Piyamitr Sritara

Ramathibodi Hospital

\section{Research Article}

Keywords: Overweight/obesity, cardiovascular events (CVEs), diabetes mellitus, hypertension, chronic kidney disease

Posted Date: September 22nd, 2021

DOl: https://doi.org/10.21203/rs.3.rs-908171/v1

License: (a) (1) This work is licensed under a Creative Commons Attribution 4.0 International License. Read Full License 


\section{Causal Relationship of Overweight/Obesity on Cardiovascular Events Through Risk Factors:}

\section{Multiple Mediation Analysis}

Thosaphol Limpijankit ${ }^{1}$, Prin Vathesatogkit ${ }^{1}$, Dujrudee Matchariyakul ${ }^{2}$, Sirichai Wiriyatanakorn ${ }^{1}$, Sukanya Siriyotha ${ }^{3}$, Ammarin Thakkinstian $^{3}$, Piyamitr Sritara ${ }^{1}$

${ }^{1}$ Division of Cardiology, Department of Medicine, Faculty of Medicine Ramathibodi Hospital, Mahidol University, Bangkok, Thailand,

${ }^{2}$ Medical and Health Office, Electricity Generating Authority of Thailand, Bangkruay, Nonthaburi 11130, Thailand

${ }^{3}$ Section for Clinical Epidemiology and Biostatistics, Faculty of Medicine Ramathibodi Hospital, Mahidol University, Bangkok, Thailand.

Toral word count: 5,044

Running title: Causal relationship of overweight/obesity on cardiovascular events

Disclosure: No relationships with industry

Corresponding author

Thosaphol Limpijankit, Division of Cardiology, Department of Medicine, Faculty of Medicine

Ramathibodi Hospital, Mahidol University 270 Rama VI Road, Ratchathewi, Bangkok, Thailand 10400

Tel.: +66-81-813-0295; Fax: +66-2-201-1660 E-mail: thosaphol.lim@mahidol.ac.th 


\section{Abstract}

Background: Overweight/obesity is associated with cardiovascular events (CVEs) and premature death. This study aimed to find the causal pathways between overweight/obesity and CVEs through risk factors in a general adult population.

Methods: A total of 7,921 employees of the Electricity Generating Authority of Thailand (EGAT) were enrolled during 2007-2009. Baseline characteristics and blood test results were collected. Body mass index $(\mathrm{BMI}) \geq 23 \mathrm{~kg} / \mathrm{m}^{2}$ by WHO criteria for Asians was defined as overweight/obesity. A mediation analysis was applied to assess potential causal pathways. BMI $\geq 23 \mathrm{~kg} / \mathrm{m}^{2}$ was considered as an independent variable, whereas diabetes mellitus (DM), hypertension (HT), and chronic kidney disease (CKD) were considered as mediators, and CVEs (i.e., fatal and non-fatal CAD or stroke) were considered as the outcomes.

Results: The prevalence of $\mathrm{BMI} \geq 23 \mathrm{~kg} / \mathrm{m}^{2}$, DM and HT, and CKD were $62.7 \%, 7.8 \%, 28.1 \%$, and $11.8 \%$ respectively. During an average of $17.2 \pm 5.5$ years follow-up, BMI $\geq 23 \mathrm{~kg} / \mathrm{m}^{2}$ subjects compared with those with lower BMIs more frequently developed CVEs $(9.4 \%$ vs $6.2 \%, p<0.001)$. The effects of $\mathrm{BMI} \geq 23 \mathrm{~kg} / \mathrm{m}^{2}$ on CVEs was significantly mediated indirectly through DM and HT with significant ORs of $1.61(1.34,2.09)$ and $1.57(1.39,1.80)$, respectively. The indirect effect of CKD on CVEs was significantly increased if mediated through DM $\rightarrow$ HT or HT [ORs of $1.17(1.09,1.32)$ and $1.20(1.10$, 1.32), respectively].

Conclusion: Subjects with overweight/obesity were prone to develop CVEs which was mediated indirectly through DM and HT. The effect of CKD on CVEs was small but enhanced if it occurred as a complication of DM or HT.

Key word: Overweight/obesity, cardiovascular events (CVEs), diabetes mellitus, hypertension, chronic kidney disease

\section{Abstract word count: 250}




\section{Causal Relationship of Overweight/Obesity on Cardiovascular Events Through Risk Factors: Multiple Mediation Analysis}

\section{Introduction}

Overweight/obesity is a major public health problem worldwide, and associated with cardiovascular events (CVEs) and premature death [1,2]. Despite increased awareness, this epidemic problem continues growing with the prevalence of overweight and obesity in adults (aged 18 years or older) reaching 39\% and 13\%, respectively, as per the WHO report in 2016 [3]. People with overweight/obesity are prone to develop type 2 diabetes mellitus (T2DM), dyslipidemia, hypertension (HT), so called 'metabolic syndrome', and to experience premature atherosclerotic cardiovascular disease (CVD) and death $[4,5]$.

Dyslipidemia is associated with atherosclerotic CVD. The linkage between dyslipidemia and atherogenesis is supported by a strong correlation between low-density lipoprotein cholesterol (LDL-C) and major adverse CVEs [6]. Reduction in LDL-C improves CVD outcomes whether achieved through primary or secondary prevention [7,8]. While a causal link between dyslipidemia and CVD is well established, such a causal association between overweight/obesity and CVD remains controversial. This is important because the increasing prevalence of overweight/obesity may eventually offset the public health gains achieved by improved treatment of CVD.

Multiple evidences shows that elevated body mass index (BMI) increases the risk of T2DM and insulin resistance $[9,10]$. In overweight/obese subjects, increased amount of non-esterified fatty acids, glycerol, proinflammatory substances, and hormones are involved in the development of insulin resistance and thus T2DM development [11]. Obesity-related HT is another important pathway to increased CVEs, and previous evidence shows a strong association of BMI with HT [12, 13]. As the prevalence of overweight/obesity increases, the prevalence of HT with its associated CV risk increases as well. The pathophysiology of obesity-related HT is insulin stimulation of the sympathetic nervous system with increased renin release, increased angiotensinogen release from intra-abdominal adipocytes and increased aldosterone production, leading to increased sodium reabsorption and obstructive sleep apnea [14].

In addition to T2DM and HT, there is a well-known relationship between BMI and renal function deterioration [15]. Numerous population-based studies have shown an association between overweight/obesity and progression of chronic kidney disease (CKD) [16-18]. The possible mechanisms 
include excessive excretory load, lipid accumulation in the kidney, metabolic abnormalities in the adipose tissue, and over activation of the renin-angiotensin-aldosterone system [19]. However, the exact mechanisms through which overweight/obesity may worsen or cause CKD remain unclear. Certainly, there is a significantly higher prevalence of T2DM and HT among overweight/obese patients with CKD (both early and late stage). Some of the worsening renal function in obesity may be mediated by downstream comorbid conditions such as T2DM or HT.

As for complex mechanisms among these diseases, we hypothesized that overweight/obesity was associated with CVEs, and probably mediated through T2DM, HT or CKD. To our knowledge, no study to date has demonstrated a causal relationship between T2DM, HT or CKD in overweight/obesity subjects and CVEs. Mediation analysis offers a tool to assess the magnitude of different pathways leading to an outcome [20]. We therefore conducted this study in a general adult population using mediation analysis to explore the possibility of a causal relationship between overweight/obesity and CVEs; if found, whether it was mediated directly or indirectly through T2DM, HT, or CKD.

\section{Methods}

\section{Study design}

The Electricity Generating Authority of Thailand (EGAT) Study is a large prospective cohort study conducted in adult employees to better understand the occurrence and influence of risk factors on CVD occurrence and progression in adult Thais. This longitudinal health study is composed of three cohorts (EGAT 1, 2, and 3) with follow-up every five years; the cohort profiles are published [21]. Briefly, employees were enrolled in the Bangkok metropolitan area (EGAT 1 and 3) and at three different sites in Western and Northern Thailand (EGAT 2). The age at enrollment ranged from 35 to 54 years, to maximize the probability of a CVD event, given that the retirement age in EGAT was 55 years. A large variety of people were recruited, from illiterates working as cleaners to truck drivers, security guards, office clerks, administrators, architectures, engineers, field explorers, lecturers, lawyers, healthcare practitioners executive board members. Inclusion criteria included the asymptomatic employees with risk factors for atherosclerosis (no CVD symptoms). Exclusion criteria included i) a diagnosis CVD at baseline (either CAD or stroke) and ii) no baseline BMI measurement.

The study protocol conformed to the ethical guidelines of the 1975 Declaration of Helsinki and was approved by the Ethics Committee of the Faculty of Medicine, Ramathibodi Hospital, Mahidol University (\# COA. No. MURA2019/402). All participants provided their informed consent. 


\section{Subjects \& data collection}

A total of 7,921 subjects from the EGAT 1, 2, and 3 cohorts were enrolled during 1997-2009. Baseline characteristics, including age, sex, atherosclerosis risk factors (e.g., T2DM, HT, dyslipidemia, smoking, and alcohol), BMI ( $\left.\mathrm{kg} / \mathrm{m}^{2}\right)$ and underlying diseases were recorded. To characterize metabolic risk factors, fasting blood glucose (FPG), lipid profile [total cholesterol, high-density lipoprotein cholesterol (HDL-C), LDL-C, triglyceride (TG)] and uric acid were collected from these subjects. T2DM was defined as either an overnight FPG $\geq 126 \mathrm{mg} / \mathrm{dL}$ or taking anti-diabetic medications. HT was defined as either systolic BP (SBP) $\geq 140 \mathrm{mmHg}$ and/or diastolic BP (DBP) $\geq 90 \mathrm{mmHg}$, or taking antihypertensive medication. An estimated GFR $\left(\mathrm{mL} / \mathrm{min} / 1.73 \mathrm{~m}^{2}\right)$ was calculated based on the separate CKD epidemiology collaboration (CKD-EPI) equations for men and women [22]. CKD was defined as an $\mathrm{eGFR}<60 \mathrm{~mL} / \mathrm{min} / 1.73 \mathrm{~m}^{2}$.

\section{BMI and categorization}

In our study, BMI was calculated as weight in kilograms divided by height in meters squared $\left(\mathrm{kg} / \mathrm{m}^{2}\right)$, and was categorized according to the WHO criteria for Asians [23]: as underweight $(<18.5$ $\left.\mathrm{kg} / \mathrm{m}^{2}\right)$, normal weight $\left(18.5-22.9 \mathrm{~kg} / \mathrm{m}^{2}\right)$, overweight $\left(23-24.9 \mathrm{~kg} / \mathrm{m}^{2}\right)$, or obese $\left(\geq 25 \mathrm{~kg} / \mathrm{m}^{2}\right)$.

\section{Follow-up and clinical outcomes}

All participants were invited to take a follow-up survey in December 2014 and 2019. The primary endpoints were CVEs (i.e., fatal and non-fatal CAD; fatal or non-fatal stroke) and all-cause deaths (ACDs). CAD was defined as the presence of at least one of the following criteria: angina, acute coronary syndrome, acute MI, a significant (> 70\% diameter stenosis) lesion on coronary angiography, revascularization (PCI or $\mathrm{CABG}$ ), and documented myocardial ischemia during exercise testing. Stroke was defined as a history of ischemic or hemorrhagic stroke, or transient ischemic attack (TIA). If a subject had two CVEs, only the first event was counted. If a subject died of a CVE, this subject was counted as both a death and a CVE. Participants who lost to follow-up were linked with death databases

[maintained by the National Health Security Office (which contained hospital discharge records) and the Department of Provincial Administration of the Ministry of the Interior (contained death certificates)] to ascertain their vital status. Each cause of death was assessed by an independent adjudication committee, comprised of cardiologists and neurologists, and classified into CAD (fatal CAD or MI or sudden 
unexplained death), stroke (including ischemic, hemorrhagic and subarachnoid hemorrhage), other vascular death (e.g. heart failure, valvular heart disease or PAD), non-CAD death [i.e., malignancy, respiratory and gastrointestinal diseases, accident, sepsis, metabolic (T2DM, HT, DLP)], or unknown.

\section{Statistical Analysis}

The characteristics of patients were expressed as means ( \pm standard deviation (SD)) and percentages for continuous and categorical variables, respectively. A univariate analysis was performed to assess the relationship of each variable with CVD using t-tests and Chi-square tests for continuous and categorical variables, respectively.

Mediation analysis using a generalized structural equation model (GSEM) was applied to assess potential causal pathways as described in Figure 1. Overweight/obesity $\left(\mathrm{BMI} \geq 23 \mathrm{~kg} / \mathrm{m}^{2}\right)$ was considered as the independent variable, whereas T2DM, HT, and CKD were considered as mediators, and CVEs was considered as the outcome of interest. Three mediation and one outcome models were constructed as follows: [24-26]:

Mediation models

$$
\begin{array}{cc}
T 2 D M=a_{0}+a_{1} \text { Overweight/obesity }+\sum_{k} e_{k} z_{k} & \text { (path } \left.a_{1}\right) \\
H T=a_{0}+a_{2} \text { Overweight/obesity }+a_{3} T D M+\sum_{k} e_{k} z_{k} & \text { (path } \left.a_{2}\right) \\
C K D=a_{0}+b_{1} \text { Overweight/obesity }+b_{2} T 2 D M+b_{3} H T+\sum_{k} e_{k} z_{k} & \text { (path } \left.b_{1} b_{2} b_{3}\right)
\end{array}
$$

Outcome model:

$$
\text { CVEs }=a_{0}+\text { cOverweight/obesity }+c_{1} D M+c_{2} H T+c_{3} C K D+\sum_{k} e_{k} z_{k} ; \text { where } z_{k}=
$$
confounders

The GSEM with logit link function was used to regressed T2DM and HT on overweight/obese (called paths $\mathrm{a}_{1}$ and $\mathrm{a}_{2}$ ). Then, the CKD mediation model was constructed to regress CKD on overweight/obese, T2DM, and HT using logit link, called path $b_{1}, b_{2}$, and $b_{3}$. Next, these overweightobese and three mediators were fitted on the CVEs outcome using a logit link (path c, $\mathrm{c}_{1}, \mathrm{c}_{2}$, and $\mathrm{c}_{3}$ ). Finally, three mediation and outcome models were simultaneously constructed using the GSEM approach. Confounders whose p-values were less than 0.10 in the univariate analysis of each mediation 
and outcome models were simultaneously considered but only significant confounders were finally retained in the multivariate GSEMs.

Potential causal mediation effects were then estimated using the products of coefficients by of the final mediation and outcome GSEM models [27]; the odds ratios (ORs) of mediation effects were then estimated [28]. A bootstrap with 5000 replications was applied to estimate mediation effects along with 95\% confidence intervals (CIs) based on a bias-corrected bootstrap technique [29]. All analyses were performed using STATA version 16. A P-value of less than 0.05 was considered to be statistically significant.

\section{Results}

A total of 7,921 out of 8,028 participants had baseline BMI and were then used for analysis. Distribution of baseline characteristics of participants grouped by BMI (i.e., BMI $<23 \mathrm{~kg} / \mathrm{m}^{2}$ and $\geq 23 \mathrm{~kg} / \mathrm{m}^{2}$ ) are summarized in Table 1 . Participants with overweight/obesity were more frequently male, T2DM, HT, CKD, ex/current smokers, ex/current drinkers and had higher levels of LDL-C, triglyceride and uric acid, but lower HDL-C. During an average of 17.2 \pm 5.5 years follow-up, overweight/obese participants developed CVEs (via both CAD and stroke) more frequently than did those nonoverweight/obese (Table 2). However, frequency of ACD, death from cancer and CAD were not significantly different between the two groups.

\section{Relationships between risk factors and BMI}

To find factors associated with T2DM, HT, CKD and CVEs, univariate regression analyses were performed (see Table 3 ). BMI $\geq 23 \mathrm{~kg} / \mathrm{m}^{2}$, older age and ex/current smoking were three common factors and associated with T2DM, HT, CKD and CVEs. For other factors, there was some inconsistency of association. Male gender, HDL-cholesterol and triglyceride were each associated with T2DM, HT and CVEs, but not with CKD. Ex or current drinking was associated with T2DM, HT, CKD, but not with CVEs. LDL-cholesterol was associated with T2DM and CKD, but not with HT nor CVEs. Uric acid was associated with HT, CKD and CVEs, but not with T2DM.

Multivariate GSEMs were constructed, simultaneously considering BMI $\geq 23 \mathrm{~kg} / \mathrm{m}^{2}$ along with these covariates (see Table 4 ). After adjusting covariables, BMI $\geq 23 \mathrm{~kg} / \mathrm{m}^{2}$ was significantly associated with T2DM, HT and CVEs but not for CKD, with the corresponding estimated ORs (95\% CI) of 1.68 $(1.39,2.04), 1.76(1.57,1.97)$, and $1.34(1.10,1.62), 1.09(0.938,1.269)$, respectively. This could be 
interpreted as overweight/obese participants being $1.68,1.76$, and 1.34 times more likely to develop T2DM, HT, and CVEs than subjects with non-overweight/obese.

In addition, participants with T2DM were $2.48(2.09,2.95)$ and $2.5(1.99,3.16)$ times more likely to develop HT and CVEs, respectively, whereas the risk for developing CKD was not significant [OR of $1.106(0.863,1.418)]$. Participants with HT were $1.6(1.36,1.84)$ and $2.2(1.86,2.64)$ times more likely to develop CKD and CVEs, respectively. Participants with CKD had a significant association with having a CVE [estimated OR of $2.01(1.62,2.49)]$. Other factors that associated with an increased risk of CVEs were age $\geq 60$, male gender, and being an ex/current smoker. As noted, high HDL and ex/current alcohol drinking were protective factors for CVEs.

\section{Mediation analysis}

Indirect effects of $\mathrm{BMI} \geq 23 \mathrm{~kg} / \mathrm{m}^{2}$ on CVEs through T2DM, HT and CKD were further estimated using a bootstrapping analysis with 5000 replications, see Table 5. The three mediation pathways that yielded the highest ORs were $\mathrm{BMI} \geq 23 \mathrm{~kg} / \mathrm{m}^{2} \rightarrow \mathrm{T} 2 \mathrm{DM}, \mathrm{BMI} \geq 23 \mathrm{~kg} / \mathrm{m}^{2} \rightarrow \mathrm{HT}$, and $\mathrm{BMI} \geq 23 \mathrm{~kg} / \mathrm{m}^{2}$ $\rightarrow \mathrm{T} 2 \mathrm{DM} \rightarrow \mathrm{HT}$ with the ORs of $1.62(1.34,2.09), 1.57(1.39,1.80)$, and $1.46(1.25,1.78)$, respectively. This could be interpreted as overweight/obese participants being about $1.34(1.11,1.67)$ times more likely to develop CVEs, and their risks being increased to 1.62, 1.57 and 1.46 if they had T2DM, HT, and T2DM plus HT, respectively. Interestingly, the effects of BMI $\geq 23 \mathrm{~kg} / \mathrm{m}^{2}$ on CVEs that were mediated through $\mathrm{CKD}$ and T2DM $\rightarrow \mathrm{CKD}$ were insignificant, with ORs of $1.063(0.962,1.195)$ and $1.037(0.956,1.149)$, respectively. However, the effect of overweight/obese on CVEs through HT and CKD would have reached significance if mediated through HT (BMI $\geq 23 \mathrm{~kg} / \mathrm{m}^{2} \rightarrow \mathrm{HT} \rightarrow \mathrm{CKD}$ ) or T2DM $\left(\mathrm{BMI} \geq 23 \mathrm{~kg} / \mathrm{m}^{2} \rightarrow \mathrm{DM} \rightarrow \mathrm{HT} \rightarrow \mathrm{CKD}\right)$, which yielded ORs of $1.20(1.10,1.32)$ and $1.16(1.08,1.32)$, respectively.

Effects of overweight/obesity on CVEs contributed through T2DM, HT, and T2DM plus HT were $29.1 \%, 27.2 \%$, and $18.6 \%$, respectively. In addition, the effects of overweight/obesity contributed through CKD alone was 3.0\%; however, effects contributed through $\mathrm{HT} \rightarrow \mathrm{CKD}$ or DM $\rightarrow \mathrm{HT} \rightarrow \mathrm{CKD}$ were $9.0 \%$ or $7.5 \%$, respectively. As noted, the direct effect of overweight/obesity on CVEs was found to be $14.3 \%$ of the contribution effect.

\section{Discussion}


We explored the potential causal relationship between overweight/obesity and CVEs, using known risk factors (including T2DM, HT and CKD) as mediators based on a 17-year longitudinal cohort study. The results from our mediation analysis suggested that overweight/obesity itself increased the risk of CVEs about $34 \%$, and the risks increased further to $57 \%, 62 \%$ and $46 \%$ if mediated through HT, T2DM, and T2DM puls HT, respectively.

A WHO panel reported that Asian populations are at risk for T2DM and CVD at lower BMIs than the standard WHO criteria of a BMI exceeding 25 [23]. The American Diabetes Association recently recommended that testing for T2DM should be considered for all Asian American adults with a BMI of $\geq 23$ [30]. This cut-off is consistent with a previous report from Japan that a BMI $\geq 23$ is a risk factor for insulin resistance and T2DM [10]. Based on these sources, we used a BMI $\geq 23$ as a definition of overweight/obesity in our cohort.

The prevalence of overweight/obesity in our study was quite significant, nearly two-thirds of participants. The majority of these overweight/obesity participants were middle-aged males; they more frequently had atherosclerotic risk factors (T2DM, HT, CKD, ex/current smoking or alcohol use, dyslipidemia and hyperuricemia). This cluster of risk factors, the so-called 'metabolic syndrome', are positively associated with incidence of CVEs and all-cause deaths [31]. During the long-term follow-up, the overweight/obesity subjects in our study developed CVEs (both non-fatal CAD and non-fatal strokes) more frequently than other subjects. These findings are similar to the previous reports of higher CVEs in overweight/obesity populations $[2,32]$. Unlike the previous literatures, there was no significantly increase in CAD and cancer deaths in our overweight/obesity population [33]. This difference raises questions that will require further follow-up of the cohort to resolve.

\section{Relationship between overweight/obesity and CVEs through mediators}

The clinical prognosis of participants with overweight/obesity ranges from mild to severe. In fact, some overweight/obese people never develop a CVE nor premature death. As many as $25 \%$ of obese individuals are classified as "metabolically healthy" suggesting that increased body weight alone is not sufficient to cause any adverse events [34]. Such individuals usually are mildly obese, young and without complication. Our aim was to find causal relationships between overweight/obesity and CVEs, whether mediated directly or indirectly through the most likely clinical risk factors.

Besides dyslipidemia and smoking which have well-established correlations with CVEs, three other major risk factors that potentially have causal links between overweight/obesity and CVEs are 
T2DM, HT and CKD. After adjusting for confounders, we found that these three risk factors still mediated an increased in the effect of overweight/obesity on CVE risk. As we mentioned previously, overweight/obesity results in complex metabolic abnormalities and may cause a high incidence of T2DM, HT and CKD. If these chronic conditions are not modified, CVEs and premature death can occur.

Behavior, environment, and genetic factors all have roles in causing people to be overweight and obese. Duration and severity of overweight/obesity, and concurrent control measures, all influence the occurrence of complications. A majority of patients are unsuccessful at changing their life-style and continue their unhealthy habits. Our study showed that the strongest mediators associated with CVEs are T2DM and HT. Subjects with overweight/obesity were more likely than those without to develop T2DM, HT, or T2DM plus HT, resulting in increased development of CVEs by 1.62 times, 1.57 times, and 1.46 times, respectively. CKD was not a strong mediator causing CVEs in contrast to T2DM and HT. It is possible that our study population included patients with early stage CKD (eGFR $<60$ $\mathrm{mL} / \mathrm{min} / 1.73 \mathrm{~m}^{2}$ ) which had less impact on CVEs occurrence. However, if CKD occurs as a complication in patients who have already developed T2DM plus HT, or HT alone, it increases the risk of CVEs.

\section{Direct effect of overweight/obesity on CVEs}

As noted, the direct effect of overweight/obesity itself on CVEs is about 1.34 times more than subjects with normal body weight, without mediation through T2DM, HT or CKD. This unidentified mechanism might be explained by genetics, inflammatory processes, hyperuricemia or decreased HDL$\mathrm{C}$ level. There is evidence that accumulation of visceral fat mass increases the production of proinflammatory cytokines and adipokines, and can promote the formation of atherosclerotic plaques [35, 36]. Moreover, obesity tends to increases the risk for CVEs, by increasing macrophage infiltration and instability of plaques $[37,38]$. Hyperuricemia is another confounding factor that is reported to predict the development of atherosclerosis. As known, hyperuricemia is frequently found in obese subjects and carries a high risk of subclinical CVD [39, 40]. Patients with metabolic syndrome usually have hyperuricemia along with other clinical criteria. Elevated serum uric acid has been shown to be a predictor of coronary calcium deposition independent of conventional CV risk factors [41]. This finding suggests that serum uric acid might be a part of mechanisms which increase the risk of CVEs in overweight/obesity populations. 
In addition, obesity is an important risk factor for decreased HDL-C levels, which predisposes to CVD [42]. There is evidence that intra-abdominal visceral fat deposition is an important negative correlate of HDL-C level [43]. HDL reduces coronary atherosclerosis by decreasing the expression of adhesion molecules on endothelial cells and thereby reducing inflammation, and by inhibiting the oxidation of LDL-C. The HDL-C level is somewhat difficult to increase, since it is mostly determined by genetics. In current practice, decreasing of LDL-C using statins and exercise is the only way to counterbalance this abnormality.

\section{Clinical implication}

The implication of this mediation analysis in terms of prevention is to encourage overweight/obesity patients to reduce their BMI to prevent early onset T2DM and HT, and also possibly to slow renal function deterioration and increase life expectancy. Early detection of all conventional risk factors and then modifying behavior and changing life-style are appropriate ways to reduce CVEs. These measures decrease disease progression and likelihood of premature death. The term of 'metabolically healthy' overweight/obesity seems to not be absolutely correct, because in our study, subjects with BMI $\geq 23 \mathrm{~kg} / \mathrm{m}^{2}$ also carry some risk of CVEs, even in the absence of T2DM, HT and CKD.

\section{Strength and limitation}

The strengths of this study include its prospective nature, large population and long duration of follow-up. Also, all clinical events were reviewed and verified by an expert committee. The EGAT project is still continuing. Its follow-up surveys every five years will continue to document clinical outcomes and thus enable the answering of some open questions. Also, our analysis included all confounding factors which might impact on CVEs and adjusted for these factors as much as possible.

Several limitations of this study needed to be mentioned. First, the association study between overweight/obesity and CVEs relies on the BMI at a single point which makes it difficult to adequate address bias associated with reverse causality. Second, the study population was enrolled only from among EGAT employees who were mostly middle aged with higher socioeconomic status and education than the general population. Our findings thus may not be generalized to those of younger age nor for the whole population. In addition, this study did not examine whether concurrent treatment or intervention with life-style modifications during the follow-up had any effect on the occurrence of 
CVEs. Lastly, the BMI that we used to group participants was at the first day of survey. Participant's BMI may have evolved during the follow-up period.

\section{Conclusions}

Overweight/obesity is a major health problem in Thailand and likely a cause of CVE occurrences. Participants with overweight/obesity trended to develop T2DM and HT leading to development of CVEs during long-term follow-up. Although effects of overweight/obese through CKD seems to have been minimal, it may be enhanced if it occurred as a complication of DM and HT. It is important that healthy overweight/obesity people also reduce their BMI to prevent T2DM and HT, increase likelihood of remaining free of disease and prevent premature death. Concurrent reduction in the magnitude of all conventional risk factors is the key for successfully reducing the occurrence of CVEs.

\section{Acknowledgments}

This work was supported by a Cooperative Research Network scholarship, the Project for Higher Education Research Promotion and National Research University Development, Office of the Higher Education Commission, Ministry of Education, Thailand, and The Thailand Research Fund. We also would like to express our appreciation to all research staff, especially Ms. Nisakorn Thongmung, for providing subject data and helpful collaboration.

\section{Conflict of Interest}

All authors declare that they have no conflicts of interests.

\section{Reference:}

1. Van Gaal LF, Mertens IL, De Block CE (2006) Mechanisms linking obesity with cardiovascular disease. Nature 14:875-80. https://doi.org/10.1038/nature05487

2. Hubert HB, Feinleib M, McNamara PM, Castelli WP (1983) Obesity as an independent risk factor for cardiovascular disease: a 26-year follow-up of participants in the Framingham Heart Study. Circulation 67:968-77. https://doi.org/10.1161/01.cir.67.5.968

3. World Health Organization (2021) Obesity and overweight: Key facts. 
https://www.who.int/news-room/fact-sheets/detail/obesity-and-overweight

4. Wilson PWF, D’Agostino RB, Parise H, et al (2005) Metabolic syndrome as a precursor of cardiovascular disease and type 2 diabetes mellitus. Circulation 112:3066-3072. https://doi.org/10.1161/CIRCULATIONAHA.105.539528

5. McNeill AM, D. RW, J. GC, et al (2005) The Metabolic Syndrome and 11-Year Risk of Incident Cardiovascular Disease in the Atherosclerosis Risk in. Diabetes Care 28:385-390

6. Borén J, John Chapman M, Krauss RM, et al (2020) Low-density lipoproteins cause atherosclerotic cardiovascular disease: Pathophysiological, genetic, and therapeutic insights: A consensus statement from the European Atherosclerosis Society Consensus Panel. Eur Heart J 41:2313-2330. https://doi.org/10.1093/eurheartj/ehz962

7. Cholesterol Treatment Trialists' (CTT) Collaboration, Baigent C, Blackwell L, et al (2010) Efficacy and safety of more intensive lowering of LDL cholesterol: a meta-analysis of data from 170,000 participants in 26 randomised trials. Lancet (London, England) 376:1670-81. https://doi.org/10.1016/S0140-6736(10)61350-5

8. Cannon CP, Blazing MA, Giugliano RP, et al (2015) Ezetimibe Added to Statin Therapy after Acute Coronary Syndromes. N Engl J Med 372:2387-2397. https://doi.org/10.1056/nejmoa1410489

9. Sinaiko AR, Steinberger J, Moran A, et al (2005) Relation of body mass index and insulin resistance to cardiovascular risk factors, inflammatory factors, and oxidative stress during adolescence. Circulation 111:1985-1991. https://doi.org/10.1161/01.CIR.0000161837.23846.57

10. Okura T, Nakamura R, Fujioka Y, et al (2018) Body mass index $\geq 23$ is a risk factor for insulin resistance and diabetes in Japanese people: A brief report. PLoS One 13:e0201052. https://doi.org/10.1371/journal.pone.0201052

11. Al-Goblan AS, Al-Alfi MA, Khan MZ (2014) Mechanism linking diabetes mellitus and obesity. Diabetes Metab Syndr Obes 7:587-91. https://doi.org/10.2147/DMSO.S67400

12. Shihab HM, Meoni LA, Chu AY, et al (2012) Body mass index and risk of incident hypertension over the life course: The johns hopkins precursors study. Circulation 126:2983-2989. https://doi.org/10.1161/CIRCULATIONAHA.112.117333

13. Hossain FB, Adhikary G, Chowdhury AB, Shawon MSR (2019) Association between body mass index (BMI) and hypertension in south Asian population: evidence from nationally-representative surveys. Clin Hypertens 25:1-9. https://doi.org/10.1186/s40885-019-0134-8 
14. Landsberg L, Aronne LJ, Beilin LJ, et al (2013) Obesity-related hypertension: pathogenesis, cardiovascular risk, and treatment: a position paper of The Obesity Society and the American Society of Hypertension. J Clin Hypertens (Greenwich) 15:14-33.

https://doi.org/10.1111/jch.12049

15. Gelber RP, Kurth T, Kausz AT, et al (2005) Association Between Body Mass Index and CKD in Apparently Healthy Men. Am J Kidney Dis 46:871-880.

https://doi.org/10.1053/j.ajkd.2005.08.015

16. Ejerblad E, Fored CM, Lindblad P, et al (2006) Obesity and Risk for Chronic Renal Failure. J Am Soc Nephrol 17:1695-1702. https://doi.org/10.1681/ASN.2005060638

17. Kramer H, Luke A, Bidani A, et al (2005) Obesity and Prevalent and Incident CKD: The Hypertension Detection and Follow-Up Program. Am J Kidney Dis 46:587-594. https://doi.org/10.1053/j.ajkd.2005.06.007

18. Vivante A, Golan E, Tzur D, et al (2012) Body Mass Index in 1.2 Million Adolescents and Risk for End-Stage Renal Disease. Arch Intern Med 172:1644. https://doi.org/10.1001/2013.jamainternmed.85

19. Kovesdy CP, Furth SL, Zoccali C (2017) Obesity and Kidney Disease: Hidden Consequences of the Epidemic. Am J Nephrol 45:283-291. https://doi.org/10.1159/000458467

20. Lange T, Vansteelandt S, Bekaert M (2012) A simple unified approach for estimating natural direct and indirect effects. Am J Epidemiol 176:190-5. https://doi.org/10.1093/aje/kwr525

21. Vathesatogkit P, Woodward M, Tanomsup S, et al (2012) Cohort profile: The electricity generating authority of Thailand study. Int J Epidemiol 41:359-365.

https://doi.org/10.1093/ije/dyq218

22. Levey AS, Stevens LA, Schmid CH, et al (2009) A New Equation to Estimate Glomerular Filtration Rate. Ann Intern Med 150:604. https://doi.org/10.7326/0003-4819-150-9-20090505000006

23. WHO Expert Consultation (2004) Appropriate body-mass index for Asian populations and its implications for policy and intervention strategies. Lancet 363:157-163.

https://doi.org/10.1016/S0140-6736(03)15268-3

24. MacKinnon DP, Fairchild AJ, Fritz MS (2007) Mediation Analysis. Annu Rev Psychol 58:593614. https://doi.org/10.1146/annurev.psych.58.110405.085542

25. Iacobucci D (2012) Mediation analysis and categorical variables: The final frontier. J Consum 
Psychol 22:582-594. https://doi.org/10.1016/j.jcps.2012.03.006

26. Baron RM, Kenny DA (1986) The moderator-mediator variable distinction in social psychological research: Conceptual, strategic, and statistical considerations. J Pers Soc Psychol 51:1173-1182. https://doi.org/10.1037/0022-3514.51.6.1173

27. MacKinnon DP, Lockwood CM, Hoffman JM, et al (2002) A comparison of methods to test mediation and other intervening variable effects. Psychol Methods 7:83-104. https://doi.org/10.1037/1082-989X.7.1.83

28. Sobel ME (1982) Asymptotic Confidence Intervals for Indirect Effects in Structural Equation Models. Sociol Methodol 13:290. https://doi.org/10.2307/270723

29. MacKinnon DP, Warsi G, Dwyer JH (1995) A Simulation Study of Mediated Effect Measures. Multivariate Behav Res 30:41-62. https://doi.org/10.1207/s15327906mbr3001_3

30. Hsu WC, Araneta MRG, Kanaya AM, et al (2015) BMI Cut Points to Identify At-Risk Asian Americans for Type 2 Diabetes Screening: Table 1. Diabetes Care 38:150-158. https://doi.org/10.2337/dc14-2391

31. Mottillo S, Filion KB, Genest J, et al (2010) The metabolic syndrome and cardiovascular risk: A systematic review and meta-analysis. J Am Coll Cardiol 56:1113-1132. https://doi.org/10.1016/j.jacc.2010.05.034

32. Bogers RP, Bemelmans WJE, Hoogenveen RT, et al (2007) Association of overweight with increased risk of coronary heart disease partly independent of blood pressure and cholesterol levels: A meta-analysis of 21 cohort studies including more than 300000 persons. Arch Intern Med 167:1720-1728. https://doi.org/10.1001/archinte.167.16.1720

33. Abdelaal M, le Roux CW, Docherty NG (2017) Morbidity and mortality associated with obesity. Ann Transl Med 5:1-12. https://doi.org/10.21037/atm.2017.03.107

34. Bala C, Craciun AE, Hancu N (2016) Updating the concept of metabolically healthy obesity. Acta Endocrinol (Copenh) 12:197-205. https://doi.org/10.4183/aeb.2016.197

35. Piché M-E, Poirier P, Lemieux I, Després J-P (2018) Overview of Epidemiology and Contribution of Obesity and Body Fat Distribution to Cardiovascular Disease: An Update. Prog Cardiovasc Dis 61:103-113. https://doi.org/10.1016/j.pcad.2018.06.004

36. Després J-P (2012) Body fat distribution and risk of cardiovascular disease: an update. Circulation 126:1301-13. https://doi.org/10.1161/CIRCULATIONAHA.111.067264

37. De Rosa R, Vasa-Nicotera M, Leistner DM, et al (2017) Coronary Atherosclerotic Plaque 
Characteristics and Cardiovascular Risk Factors - Insights From an Optical Coherence Tomography Study. Circ J 81:1165-1173. https://doi.org/10.1253/circj.CJ-17-0054

38. Lovren F, Teoh H, Verma S (2015) Obesity and atherosclerosis: mechanistic insights. Can J Cardiol 31:177-83. https://doi.org/10.1016/j.cjca.2014.11.031

39. Lv S, Liu W, Zhou Y, et al (2019) Hyperuricemia and severity of coronary artery disease: An observational study in adults 35 years of age and younger with acute coronary syndrome. Cardiol J 26:275-282. https://doi.org/10.5603/CJ.a2018.0022

40. Wang H, Jacobs DR, Gaffo AL, et al (2013) Longitudinal association between serum urate and subclinical atherosclerosis: the Coronary Artery Risk Development in Young Adults (CARDIA) study. J Intern Med 274:594-609. https://doi.org/10.1111/joim.12120

41. Jun JE, Lee Y-B, Lee S-E, et al (2018) Elevated serum uric acid predicts the development of moderate coronary artery calcification independent of conventional cardiovascular risk factors. Atherosclerosis 272:233-239. https://doi.org/10.1016/j.atherosclerosis.2018.02.014

42. Bora K, Pathak MS, Borah P, Das D (2017) Association of Decreased High-Density Lipoprotein Cholesterol (HDL-C) With Obesity and Risk Estimates for Decreased HDL-C Attributable to Obesity: Preliminary Findings From a Hospital-Based Study in a City From Northeast India. J Prim Care Community Health 8:26-30. https://doi.org/10.1177/2150131916664706

43. Rashid S, Genest J (2007) Effect of Obesity on High-density Lipoprotein Metabolism**. Obesity 15:2875-2888. https://doi.org/10.1038/oby.2007.342 


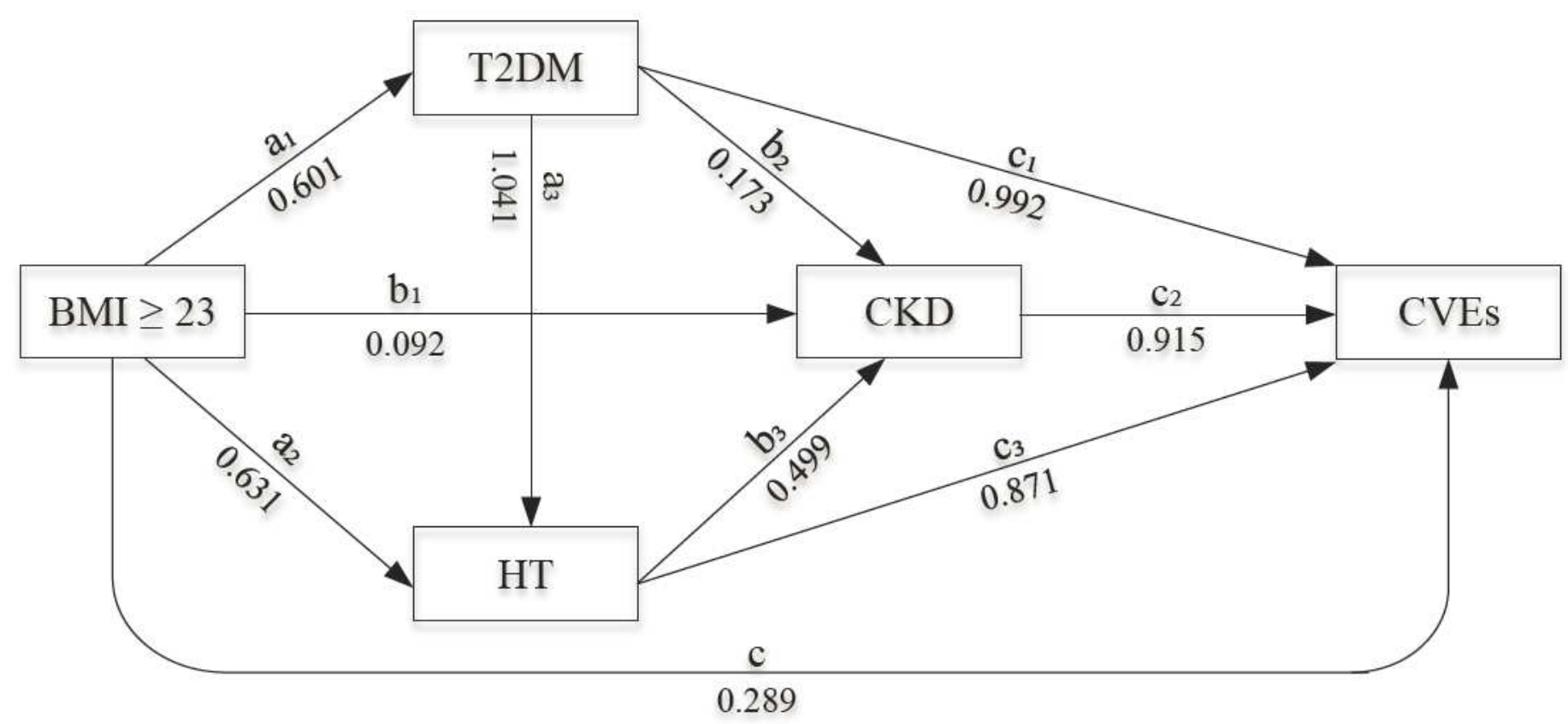

Figure 1

Causal pathway diagrams of overweight-obese and CVEs through T2DM, HT, and CKD mediators

\section{Supplementary Files}

This is a list of supplementary files associated with this preprint. Click to download.

- Table1.pdf

- Table2.pdf

- Table3.pdf

- Table4.pdf

- Table5.pdf 